\begin{abstract}
Ayse Gul Kocak Altintas*, Mehmet
Citirik and Bayram Gulpamuk

SB Ankara Ulucanlar Eye Training and Research Hospital

Dates: Received: 08 June, 2016; Accepted: 29 August, 2016; Published: 30 August, 2016

*Corresponding author: Ayse Gul Kocak Altintas, Medical Doctor, Associated Professor, Ulucanlar, Cad. No: 59 06230, Altindag, Ankara, Turkey, Tel: 90-312-3126261; Fax: 90-312-3124827; E mail: aysegulkaltintas@hotmail.com

www.peertechz.com

ISSN: 2455-1414
\end{abstract}

Keywords: Central macular thickness; Diabetes mellitus; HbA1c; Serum lipids

\section{Research Article \\ Relationship of Serum HbA1c and Fasting Serum Lipids with Central Macular Thickness in Patients with Type 2 Diabetes Mellitus}

\section{Abstract}

Background: Diabetic retinopathy, the most common retinal vascular disease, is the leading cause of new-blindness in adults during the third through sixth decades of life.

Purpose: To determine the correlation between central macular thickness (CMT) and both $\mathrm{HbA1c}$ and fasting serum lipids level in early stage non proliferative diabetic retinopathy.

Results: The patients were divided into two groups as HbA1c $>8.67 \%$ (Group I, $\mathrm{n}: 60$ ) and $\mathrm{HbA1c}$ $\leq 8.67 \%$ (Group II, $\mathrm{n:} 72$ ). The mean CMT of better eyes was $273.28 \pm 34.61 \mu \mathrm{m}$. The mean CMT of worse eye was $304.40 \pm 64.31 \mu \mathrm{m}$. According to comparison between Group I ( $\mathrm{HbA} 1 \mathrm{C}>8.67 \%)$ and Group II (HbA1c $58.67 \%$ ) the mean CMT's were not different in two groups ( $P: 0.37)$.

Main findings: $\mathrm{HbA} 1 \mathrm{c}$ level (8.76\%) were significantly higher than normal upper limits in patient even under diabetic medication. In further analyses the mean CMT's was not different in worse eye in Group I and Group II and it was not significantly different in better eyes in these two group either.

Conclusion: Even we did not observed any correlation between level of HbA1c and the degree of CMT statistically in either worse or better eye, result of our study showed that patient had increased macular thickness even early stage of NPDR while having significantly higher HbA1c even under systemic medication.

Brief Summary: The purpose of the current study was to determine the correlation between diabetic macular edema and both $\mathrm{HbA1c}$ and fasting serum lipids in early stage non proliferative diabetic retinopathy. We did not observed any correlation between level of HbA1c and the degree of central macular thickness (CMT) statistically in either worse or better eye. Our results may indirectly evidence of insufficient metabolic treatment can cause macular edema prior to other retinal signs.

Potential implications: Systemic risk factor for follow-up of macular edema secondary to diabetes mellitus.

\section{Introduction}

Diabetes mellitus (DM) is a serious metabolic syndrome associated with hyperglycemia due to either insulin resistance or insufficiency. This chronic disease affects many organs and leading to severe complications one of which is diabetic retinopathy (DR) $[1,2]$. DR is the most common micro vascular complications of long-standing DM. Various diabetes risk scores composed of both laboratory and non-laboratory parameters have been developed. But plasma glycosylated hemoglobin known as Hemoglobin Alc (HbAlc) is as an index of average glycemic control over the previous 2-3 months are currently used both for monitoring and diagnosing for patients with diabetes mellitus [3,4]. Increased $\mathrm{HbA} 1 \mathrm{c}$ concentration is the most important risk factor for developing DM complications including DR which is presented with retinal bleeding, vascular occlusion, ischemia, neovascularization, edema of retina or macula. Diabetic macular edema (DME) remains a major cause of visual impairment and blindness in diabetic patients. Increased HbA1c concentration, elevated serum lipids, uncontrolled high blood pressure, presence of nephropathy and/or neuropathy are the most important risk factor for predicting DR complications [5-9].

Optical coherence tomography (OCT) is a noninvasive imaging modality that enabled to in vivo visualize of biological tissues in human eyes. By OCT examination multiple thin slices, laminar sheet structural images of morphologic changes, including vitreomacular traction, diffuse retinal thickening, intraretinal cystic changes, subretinal fluid can be easily detected. The central macular thickness (CMT) has been the most commonly used parameter to evaluate DME, in clinical follow up and research trials [10,11].

The purpose of the current study was to determine the correlation between diabetic macular edema, examined by OCT, and both $\mathrm{HbA1c}$ and fasting serum lipids.

\section{Materials and Methods}

The presented study protocol and informed consent forms were reviewed and approved by the local medical Ethics Committee of Diskapi Training and Research Hospital. This study was designed in accordance with the Declaration of Helsinki. 
A retrospective analysis was conducted on 264 eyes of 132 patients with type $2 \mathrm{DM}$ who had admitted to retina clinic in Ulucanlar Eye Education and Research hospital for routine ophthalmologic examination for the first time. A completeophthalmologic examination including visual acuity and intraocular pressure measurement, anterior segment examination, dilated fundus examinations were performed to all patients. The presence of DM in all patients had been confirmed by the corresponding internal medicine department. The status of retinopathy was assessed by fundus photography, fluorescein angiography and also by optical coherence tomography. Early Treatment of Diabetic Retinopathy Study criteria were used to define stages of diabetic retinopathy. According to fundus findings patient who diagnosed Non Proliferative Diabetic Retinopathy (NPDR) included in this study. Patient with proliferative DR, eyes had received any treatment such as laser photocoagulation or intravitreal injections were excluded. Patients having history of any other ocular disease including any other retinal disease, glaucoma, uveitis and history of trauma or ocular surgery as well as patients being under control of another ophthalmology clinic for DR were not included.

Data on duration of diabetes, presence of other co-morbidities including hypertension, nephropathy, neuropathy, which are known to be associated with metabolic syndromes and any other systemic diseases were also checked. Types of diabetic treatment and antihypertensive or lipid lowering medications were obtained from each patient. For statistical evaluation data on age, gender, fasting serum lipid, high-density lipoprotein cholesterol (HDL), low-density lipoprotein cholesterol (LDL), triglyceride (TG), plasma HbA1c and central macular thickness (CMT) were obtained from each patient.

A SD-OCT volume scan $(20 \times 20$ with 49 horizontal sections, ART 15) including en face images and macular mapping image obtained with HRA2 (Heidelberg Retina Angiograph-Optical Coherence Tomography, Heidelberg Engineering, Heidelberg, Germany) of the macula was performed for each study eye. Retinal thickness in the Early Treatment Diabetic Retinopathy Study (ETDRS) subfields was analyzed by the retinal thickness (RT) map analysis protocol and CMT was determined. High quality images were obtained by eliminating motion artifacts using eye tracker system. Retinal findings including presence or absence of any other retinal pathology such as age related macular disease, hereditary maculopathy, macular cystic changes or diffuse macular thickening were evaluated by same observer and CMT were measured and recorded for statistical evaluation.

Statistical analyses of data were performed using with the Statistical Package for the Social Sciences software (SPSS Inc., Chicago, IL, USA) version 15 . The variables were evaluated by analytical statistical methods. Kolmogorov-Smirnov/Shapiro-Wilks test used to assess normality of variables. Descriptive statistics were expressed as median, minimum-maximum, for non-normally distributed variables. In order to evaluate the correlations between non-normally distributed variables, the correlation coefficients and their significance were calculated using the Spearman's correlation test. Mann-Whitney U test was used for evaluation of correlation between non-normally distributed numerical variables of groups. A $\mathrm{p}$ value $<0.05$ was considered significant.

\section{Results}

In the current study, out of 132 patients 48 (36.4\%) were male and $84(63.6 \%)$ were female. The number of female patient was significantly higher than male subjects (P: 0.004). The mean age of the patients was $59 \pm 8.9$ years (range, $38-80$ years). In the female group mean age was $59 \pm 7.8$ years (range, 38-74 years) while in male patient it was $56.5 \pm 8.8$ (range, $39-80$ years). The mean age of female patient was significantly higher than male patient. (P: 0.04)

Hypertension was present in 57 (43.2 \%) patients, while nephropathy, neuropathy or any other systemic diseases were not present in any of the patients. Hypertension was found as a most frequent co-morbidity factor in diabetic patients in this study. There was also no significant difference between genders in terms of comorbidities.

The mean HbAlc was $8.67 \%$ (range, 6\%-14.6\%) which was significantly higher than the normal upper limits of testing laboratory value (6\%). According to Kolmogorov-Smirnov test, HbA1c had normal distribution (P: 0.61) thus, for further evaluation, the patients were divided into two groups as HbAlc $>8.67 \%$ (Group I, n: 60) and HbA1c $\leq 8.67 \%$ (Group II, n: 72).

No one had clinically significant macular edema (CSME) on fundoscopic evaluation. The mean CMT of all eyes was $297.12 \pm$ $101.38 \mu \mathrm{m}$; the median was $279.75 \mu \mathrm{m}$ (range, 201-550 $\mu \mathrm{m}$ ). The central macular thickness was not symmetric in most of the patients and results of Kolmogorov-Smirnov test showed that CMT was not normally distributed. For statistical evaluation, the eye with higher CMT of each patient was considered as a worse and the eye with lower CMT of the same patient was considered as a better eye. The mean CMT of better eyes was $273.28 \pm 34.61 \mu \mathrm{m}$ and the median was $269.01 \mu \mathrm{m}$ (range, 201-449 $\mu \mathrm{m}$ ). The mean CMT of worse eye was $304.40 \pm 64.31 \mu \mathrm{m}$ and the median was $284 \mu \mathrm{m}$ (range, 219-550 $\mu \mathrm{m}$ ) (Table 1). According to these findings the CMT's were higher than our (SD-OCT) normal laboratory limits of $(250 \mu \mathrm{m})$ even in the minimally affected retinas in eyes with NPDR. All of these parameters about CMTs and HbAlc were significantly higher than that of normal reference values. On correlation analyses with Spearman's correlation test, there was not any significant correlation between the HbA1c level and CMT of all eyes (R: -0.12 P: 0.16). In subgroup analyses there was not any correlation between HbAlc level and with worse or better eyes either (R:-0.07 P: 0.40; R:-0.05 P: 0.53 respectively). According to comparison between Group I (HbAlc > 8.67\%) and Group II (HbAlc $\leq 8.67 \%$ ) the mean CMT's were not different in two groups (P: 0.37 ). In further analyses the mean CMT's was not different in worse eye in Group I and Group II and it was not significantly different in better eyes in these two group either (P: 0.24 and P: 0.15 respectively).

Table 1: Central macular thickness (CMT) values in better and worst eyes in subject with high (>8.6) and low HbA1c ( $\leq 8.6)$.

\begin{tabular}{|l|l|l|l|}
\hline CMT $(\boldsymbol{\mu m})$ & $\begin{array}{l}\text { Group I (HbA1c>8,6) } \\
(\mathbf{n}=60)\end{array}$ & $\begin{array}{l}\text { Group II (HbA1c } \mathbf{8}, \mathbf{6}) \\
(\mathbf{n = 7 2 )}\end{array}$ & P-value* \\
\hline Mean CMT & $285.7(212.5-464.5)$ & $291.45(228-456)$ & 0,372 \\
\hline CMT of better eye & $270.43(201-449)$ & $275.65(212-378)$ & 0,156 \\
\hline CMT of worse eye & $300.96(219-504)$ & $307.26(229-550)$ & 0,247 \\
\hline *Mann-Whitney U Test. & & \\
\hline
\end{tabular}


The mean HDL, LDL and TG levels were $49.46 \pm 7.8 \mathrm{mg} / \mathrm{dl}$, $111.83 \pm 11.0 \mathrm{mg} / \mathrm{dl}$, and $182.31 \pm 18.2 \mathrm{mg} / \mathrm{dl}$ respectively. Although result of Kolmogorov-Smirnov normalization test, each fasting serum lipids including HDL, LDL and TG had not normal distribution ( $\mathrm{p}: 0,00, \mathrm{p}: 0,03, \mathrm{p}: 0,00$, respectively), according to laboratory analyses of the each fasting serum lipids were in normal laboratory ranges (Table 2). Therefore any further subgroup analyses about serum lipids level and its correlation with CMT did not performed.

\section{Discussion}

$\mathrm{HbAlc}$ is as an index of average glycemic control which is currently used both for diagnosing and monitoring for patients with diabetes mellitus in most clinics. Increased $\mathrm{HbAlc}$ concentration which is a quantitative indicator that shows poor diabetic control, furthermore, it is the most important risk factor for predicting DM complications such as diabetic retinopathy or maculopathy $[1,2]$. According to different laboratory cut of point, maintaining an $\mathrm{HbAlc}$ level below $6 \%$ or $6.5 \%$ is critical to decrease the incidence of diabetic complications. DR is among the most common complications of diabetes which can result in edema of the central retina or macula. $\mathrm{DME}$ is one of the major causes of vision impairment and blindness in patients with diabetes [12-14].

The purpose of the present study was to determine the correlation between CMT and HbAlc level in early stage NPDR. Our results showed that $\mathrm{HbAlc}$ level $(8.76 \%)$ were significantly higher than normal upper limits in patient even under systemic diabetic medication. Benarous et al. [2], reported that HbA1c was 7.3 in patient without DR while it was 8.0 in eyes with DR, 8.1 in eyes presented with DME, and 8.3 in eyes with CSME. Similarly, Jew et al. [15], found that HbAlc level was significantly higher in the eyes with CSME (10.38\%) compared to that in eyes without CSME (7.8\%).

Unsal et al. [16], also found a correlation with diabetic retinopathy stage, macular involvement and the mean $\mathrm{HbA1c}$ levels which were 8.2\% in the NPDR group, 9.5\% in the eyes with PDR, and 9.0\% in DME group. Sasaki et al. [17], evaluated 74 patient with no DR and 7 patient with only minimal DR and found that HbA1c level (7.2\%) was above the normal upper limits. Cetin et al. [5], reported that HbA1c level was higher in eyes with DME (8.6\%) than in eyes without DME (7.9\%). According to multivariate analysis in Jew et al. [15], study, they showed that HbAlc levels were one of the significant risk factor for developing CSME. Even we did not observed CSME based on fundoscopic evaluation in none of our patient and we did not included the patient with PDR, all of our subjects with bilateral NPDR had increased HbAlc level similar to the other reports.

Table 2: Patients fasting serum lipid level according to Kolmogorov-Smirnov's normalization test, none of these parameters were in normal distribution. Patient's serum glycosylated hemoglobin ( $\mathrm{HbA} 1 \mathrm{c})$ level was in normal distribution tested by Kolmogorov-Smirnov's test.

\begin{tabular}{|l|l|l|l|l|}
\hline & HDL $(\mathbf{m g} / \mathbf{d l})$ & LDL $(\mathbf{m g} / \mathbf{d l})$ & TG $(\mathbf{m g} / \mathbf{d l})$ & HbA1C (\%) \\
\hline Mean $(\mathrm{mg} / \mathrm{dl})$ & 49.46 & 111.83 & 182.31 & 8.67 \\
\hline Maximum $(\mathrm{mg} / \mathrm{dl})$ & 87 & $294 \mathrm{~V}$ & 585 & 14.6 \\
\hline Minimum $(\mathrm{mg} / \mathrm{dl})$ & 25 & 28 & 54 & 6 \\
\hline Reference Value & $45-65$ & $0-130$ & $0-200$ & $4-6$ \\
\hline P Value & 0.00 & 0.03 & 0.00 & 0.61 \\
\hline
\end{tabular}

Unsal et al. [16], observed that the mean CMT $(371 \mu \mathrm{m})$ in eyes with DME was significantly higher than the eyes with PDR $(256 \mu \mathrm{m})$ and NPDR $(253 \mu \mathrm{m})$. In series by Benarous et al. [2], the mean CMTs were $236 \mu \mathrm{m}$ in eyes with DR, $250 \mu \mathrm{m}$ in eyes with DME, and 301 $\mu \mathrm{m}$ in eyes with CSME while it was only $215 \mu \mathrm{m}$ in eyes without DR evaluated with time-domain OCT (TD-OCT). Each of these studies demonstrated positive associations between diabetic stage and macular thickness. Moreover Sasaki et al. [17], reported that the mean CMT was $273 \mu \mathrm{m}$ in eyes with no (74) or minimal DR (7) which measured by SD - OCT. Their result was higher than that of other series such as even in eyes with DME reported in Benarous et al. [2], series. This difference may due to measurement quality using with different type of OCT. SD-OCT has the greater resolution which has more sensitive in evaluation and measurement than TD-OCT. The CMT in presenting study was measured by SD-OCT similar to Sasaki et al. [17], series. The mean CMT was $297.12 \mu \mathrm{m}$ which was higher than that of their series. The difference may related to diabetic stage in which each 264 eyes of 132 patients in our series had NPDR while only 7 eyes had minimal DR in Sasaki et al [17] series.

There are several conflicting reports in the literature regarding the effect of lipid profile on CMT and maculopathy. Cetin et al [5] did not observe any association with total cholesterol, TG or LDL levels and macular edema. Benarous et al. [2], showed that serum lipid levels were not correlated with both CMT and macular volume. Similarly, Sasaki et al [17] reported that with covariables adjustment each serum lipids; total cholesterol, HDL, and TG levels were not significantly associated with both CMT. In the presenting study while the mean fasting serum lipid levels were in the range of reference levels, the mean CMT were higher than the normal limits that may be indirect evidence of absence of any correlation between the macular thickness and the serum lipid profile.

As far as we know even the correlation of HbAlc levels with DM complications have evaluated by several investigators there was not any study has focused on the correlation between $\mathrm{HbAlc}$ level and CMT in eyes with naive NPDR. To quantitatively evaluated the level of macular thickness both in better and worse eye and its correlation with HbAlc in eyes with naive DR, only the patients who were admitted to ophthalmology clinic for the first time were examine. In order to minimize the effect of confounding factors related to treatment either with laser or intravitreal injections and the severity of DR, we examined only the treatment-naive eyes with minimal NPDR. Even we did not observed any correlation between level of $\mathrm{HbA1c}$ and the degree of CMT statistically in either worse or better eye of patient with DR, result of our study showed that patient had increased macular thickness even early stage of NPDR while having significantly higher HbAlc even under systemic medication. Our results may indirectly evidence of insufficient metabolic treatment can cause macular edema prior to other retinal signs. The limitation of our retrospective study is evaluation of correlation between CMT and duration of diabetes. Further studies are necessary to evaluate how both maculas are asymmetrically affected by same systemic risk factors in same subject and fine cut-off point of HbAlc for increment of CMT. 


\section{Acknowledgement}

All persons named in the Acknowledgment section have provided me with written permission to be named

\section{References}

1. Cade WT (2008) Diabetes-Related Microvascular and Macrovascular Diseases in the Physical Therapy Setting. Phys Ther 88: 1322-1335.

2. Benarous R, Sasongko MB, Qureshi S, Fenwick E, Dirani M, et al. (2011) Differential association of serum lipids with diabetic retinopathy and diabetic macular edema. Invest Ophthalmol Vis Sci 52: 7464-7469.

3. Criel M, Jonckheere S, Langlois M (2016) Evaluation of Three Hemoglobin A1c Point-of-Care Instruments. Clin Lab 62: 285-291.

4. International Expert Committee, Nathan DM (2009) International Expert Committee report on the role of the A1C assay in the diagnosis of diabetes. Diabetes Care 32: 1327-1334.

5. Cetin EN, Bulgu Y, Ozdemir S, Topsakal S, Akin F, et al. (2013) Association of serum lipid levels with diabetic retinopathy. Int J Ophthalmol 6: 346-349.

6. Hirano M, Nakanishi S, Kubota M, Maeda S, Yoneda M, et al. (2014) Low highdensity lipoprotein cholesterol level is a significant risk factor for development of type 2 diabetes: Data from the Hawaii-Los Angeles-Hiroshima study. Diabetes Investig 5: 501-506.

7. Tajunisah I, Nabilah H, Reddy SC (2006) Prevalence and risk factors for diabetic retinopathy-a study of 217 patients from University of Malaya Medical Centre. Med J Malaysia. 61: 451-456.

8. Idiculla J, Nithyanandam S, Joseph M, Mohan VA, Vasu U, et al. (2012) Serum lipids and diabetic retinopathy: A cross-sectional study. Indian J Endocrinol Metab 16: 492-494.
9. Sachdev N, Sahni A (2010) Association of systemic risk factors with the severity of retinal hard exudates in a north Indian population with type 2 diabetes. J Postgrad Med 56: 3-6.

10. Buabbud JC, Al-latayfeh MM, Sun JK (2010) Optical coherence tomography imaging for diabetic retinopathy and macular edema. Curr Diab Rep 10: 264269.

11. Tan CS, Chew MC, Lim LW, Sadda SR (2016) Advances in retinal imaging for diabetic retinopathy and diabetic macular edema. Indian J Ophthalmol 64: 76-83.

12. Lee WJ, Jang S, Lee SH, Lee HS (2016) Correlation between the severity of diabetic peripheral polyneuropathy and glycosylated hemoglobin levels: a quantitative study. Ann Rehabil Med 40: 263-270.

13. Matthews DR, Stratton IM, Aldington SJ, Holman RR, Kohner EM (2004) Risks of progression of retinopathy and vision loss related to tight blood pressure control in type 2 diabetes mellitus: UKPDS 69. Arch Ophthalmol 122: $1631-1640$.

14. ADVANCE Collaborative Group, Patel A, MacMahon S, Chalmers J, Neal $B$, et al. (2008) Intensive blood glucose control and vascular outcomes in patients with type 2 diabetes. N Engl J Med 358: 2560-2572.

15. Jew OM, Peyman M, Chen TC, Visvaraja S (2012) Risk factors for clinically significant macular edema in a multi-ethnics population with type 2 diabetes. Int J Ophthalmol. 5: 499-504.

16. Unsal E, Eltutar K, Zirtilolu S, Dinçer N, Ozdoan Erkul S, et al. (2014) Choroidal thickness in patients with diabetic retinopathy. Clin Ophthalmol 8 637-642.

17. Sasaki M, Kawashima M, Kawasaki R, Uchida A, Koto T, et al. (2014) Association of serum lipids with macular thickness and volume in type 2 diabetes without diabetic macular edema. Invest Ophthalmol Vis Sci 55: 1749-1753.

Copyright: (c) 2016 Kocak Altintas AG, et al. This is an open-access article distributed under the terms of the Creative Commons Attribution License, which permits unrestricted use, distribution, and reproduction in any medium, provided the original author and source are credited. 